\title{
Development of Aroma Massage Oil for Relieving Muscle Pain and Satisfaction Evaluation in Humans
}

\author{
Tapanee Hongratanaworakit*, Sukanya Soontornmanokul, Phattarapond Wongareesanti \\ Faculty of Pharmacy, Srinakharinwirot University, Rangsit-Nakhon-Nayok Road, Nakhon-Nayok, 26120, Thailand.
}

\section{ARTICLE INFO \\ Article history: \\ Received on: 17/01/2018 \\ Accepted on: 23/02/2018 \\ Available online: 29/04/2018}

\section{Key words:}

Aromatherapy massage

oil, muscle pain, odor

preference, odor acceptance,

irritation, sensitization.

\begin{abstract}
The objective of this study was to develop aroma massage oil containing natural oils for relieving muscle pain and evaluate users' satisfaction. In addition, the skin irritation and skin sensitization of products were tested by using the Kligman maximization method. Seven essential oils were used as an active ingredient in the formula. Six carrier oils were used as a carrier oil in the formula. Tests of odor preference and odor acceptance of products were investigated in sixty-one consumers. The products were evaluated the preliminary physical stability in terms of color, smell, rancidity, viscosity, and precipitation, for a period of eight weeks. In addition, thirty consumers participated in the satisfaction evaluation. The results showed that consumers gave the most preference and acceptance to a blended essential oil preparation 1 (BEOP1) which composed of rosemary, lavender, patchouli, eucalyptus, and peppermint oils. The consumers gave an overall satisfied with carrier oil formulas which consisted of sweet almond, grape seed, avocado, jojoba oils, and macadamia oils. A massage oil formula which composed of BEOP1, vitamin E acetate, isopropyl myristate, and carrier oils gave the best physical characteristics. Moreover, developed massage oil remained stable for 8 weeks. No skin irritation and no skin sensitization were observed after volunteers applied to developed massage oil on the inner forearm for two weeks. Therefore, it could be suggested that developed massage oil was safety.
\end{abstract}

\section{INTRODUCTION}

Presently, muscle pain is prevalent in all ages. Muscle pain causes for many reasons, such as improper use of muscles, use of excessive muscle, too many exercises. In addition, anxiety or stress results in muscle pain as well. Modern medicine approaches to treat and relieve muscle pain using analgesic and antiinflammatory drugs. Such drugs are mostly synthetic chemicals which may cause adverse drug reactions such as drowsiness, stomach irritation. For this reason, some consumers turn to use more natural products to relieve muscle pain. Treatment of muscle pain with essential oils is a choice of alternative medicine, which are very popular and can be observed from the expansion of the market for health products containing oil, aroma products, and spa products (Price and Price, 2003). Moreover, essential oils are derived from natural products which are safer than synthetic drugs.

\footnotetext{
${ }^{*}$ Corresponding Author

Tapanee Hongratanaworakit, 63 Moo 7, Faculty of Pharmacy, Srinakharinwirot University, Rangsit-Nakhon-nayok Road, Nakhon-Nayok, 26120, Thailand.E-mail: tapanee@g.swu.ac.th
}

Essential oils are not only used to relieve muscle pain but also help to relax or stimulate the mind (Cooksley, 1996). However, use of essential oil alone may require high doses to achieve effectiveness for relieving muscle pain. Moreover, the consumer may not like the smell of the single oil. Therefore, aromatherapy blending products seem to be the choice of consumers to receive product smells pleasant. In addition, using blended essential oils together would provide a synergistic effect better than using essential oil alone (Cooksley, 1996; Damain and Damain, 1995).

Some studies have shown that essential oils were used for muscle pain and inflammation such as eucalyptus, rosemary, geranium, patchouli, clary sage, lavender, sweet marjoram, camphor, tea tree, black cumin, peppermint, and citrus oils (Albert and Steven, 1996; Golab and Skwarlo-Sonta, 2007; Hajhashemi et al., 2004; Hong and Shellock, 1991; Lawless, 1999; Lis-Balchin, 2006; Tekeoglu et al., 2006). Our past study (Koonlaboot and Hongratanaworakit, 2015) demonstrated that blending essential oil preparation (petty patent number 10269) comprising eucalyptus, rosemary, patchouli oil, and sweet marjoram, relieve muscle pain in volunteers. The study design was a quasi-experimental study with 
one group pretest-post-test. Thirty-five volunteers participated in this study. The volunteers assessed muscle pain before and after applying the product as well as rated satisfaction with the use of such product. The severity of pain was assessed by a numerical pain scale (0-10\%). The results showed that volunteers felt muscle pain decreased significantly after using the product $(\mathrm{p}<0.001)$. Overall satisfaction with the product was in high level. However, the opinion after using the product showed that the product had sticky and oily on the skin too much which made the users low satisfaction. Therefore, it should improve the ability to penetrate the skin and product's oily to achieve maximum satisfaction.

Essential oils may be used alone or blend two, three or more oils together to match an individual's physical and emotional needs. Good blends usually combine all three notes. Top notes (such as eucalyptus and peppermint) are highly volatile and give the initial scent. Middle notes (such as rosemary, lavender, ginger, and sweet marjoram) round out the blend and last a little longer. Base notes (such as patchouli and sandalwood) are longlasting and acts as a fixative. Generally, the blends composed of top note $(20-40 \%)$, middle note $(50-70 \%)$, and base note $(10 \%)$ (Hudson, 1999; Wildwood, 2000). In this study, we selected seven essential oils which had been reported an analgesic or/and antiinflammation effects (Gobel et al., 1994; Juergens et al., 1998a; Juergens et al., 1998b; Larry et al., 2009; Lis-Balchin, 2006; Narishetty and Panchagnula, 2005; Price and Price, 2003; Romano and Stiller, 1994; Rutledge and Jones, 2007; Silva et al., 2003). The eucalyptus and peppermint oils were used as a top note. The rosemary, sweet marjoram, lavender, and ginger oils were used as a middle note. The patchouli oil was used as a base note.

In general, carrier oils are used to dilute essential oils for use in aromatherapy massage and to make beauty preparations. Containing vitamins, proteins, and minerals, they are highly effective moisturizers and provide many of the nutrients that the skin needs to keep it smooth. Basic carrier oil or all-purpose carrier oils such as sweet almond, apricot kernel, peach kernel, grape seed, sunflower can be used alone or enriched with special carrier oils. Special carrier oils such as avocado, sesame, rose hip, wheat germ can be added to basic carrier oils to improve penetration of the skin, nourish dry, dehydrated skin, or extend the life of an oil blend (Hudson, 1999). In this study, we selected three basic carrier oils, i.e., sweet almond, apricot kernel, grape seed, and three special carrier oils, i.e., avocado, jojoba, macadamia.

The objective of the study was to develop aroma massage oil containing natural oils for relieving muscle pain and evaluate users' satisfaction. In addition, the skin irritation and skin sensitization of the product were tested by using the Kligman maximization method to ensure that the product does not cause irritation and allergic to consumers when products are used in both the short and long terms. The data obtained from this study will be useful for aromatherapy products to relieve muscle pain and can be used as a body of knowledge in further research related to the formulation development of natural products.

\section{MATERIALS AND METHODS}

Essential oils, i.e., eucalyptus (Eucalyptus globulus Labill.), peppermint (Mentha piperita L.) rosemary (Rosmarinus officinalis L.), sweet marjoram (Origanum marjorana L.), lavender (Lavandula angustifolia Mill.), ginger (Zingiber officinalis), patchouli (Pogostemon cablin (Blanco) Benth) were purchased from Thai-China Flavors and Fragrances Industry Co., Ltd., Thailand. Carrier oils, i.e., sweet almond, grapeseed, apricot, avocado, jojoba, macadamia, were purchased from Make Scents Co., Ltd., Thailand.

\section{Formulation of blended essential oil preparations (BEOPs)}

BEOPs were prepared in a volume ratio, as shown in Table 1. BEOP 1 was composed of rosemary: lavender: patchouli: eucalyptus: peppermint oils (5:5:1:4:5). BEOP 2 was composed of rosemary: lavender: patchouli: eucalyptus: ginger oils $(5: 5: 1: 4: 5)$. BEOP 3 was composed of rosemary: lavender: patchouli: eucalyptus: sweet marjoram oils (5:5:1:4:5). BEOP 4 was composed of rosemary: lavender: patchouli: ginger: peppermint oils $(5: 5: 1: 4: 5)$. BEOP1 prepared as following: pipette $5 \mathrm{~mL}$ of rosemary, $5 \mathrm{~mL}$ of lavender, $5 \mathrm{~mL}$ of peppermint, $4 \mathrm{~mL}$ of eucalyptus, and $1 \mathrm{~mL}$ of patchouli oils in a light-resistant bottle. Then close tightly and shake well until homogeneous. Kept in a cool place until use. Other BEOPs were prepared in the same procedure as BEOP1 but changing the amount and type of the essential oils.

Table 1: Blended essential oil preparations (BEOPs).

\begin{tabular}{ccccc}
\hline Preparation $(\mathbf{m L})$ & BEOP1 & BEOP2 & BEOP3 & BEOP4 \\
\hline Rosemary & 5 & 5 & 5 & 5 \\
Lavender & 5 & 5 & 5 & 5 \\
Patchouli & 1 & 1 & 1 & 1 \\
Eucalyptus & 4 & 4 & 4 & - \\
Peppermint & 5 & - & - & 5 \\
Ginger & - & 5 & - & 4 \\
Marjoram & - & - & 5 & - \\
\hline
\end{tabular}

\section{Odor preference and odor acceptance tests}

Because of difference in satisfaction of consumers, tests of odor preference and odor acceptance to BEOPs were carried out with consumers. Sixty-one healthy consumers took part in the odor preference and odor acceptance tests. They were fully briefed, given written informed consent to all aspects of the study (Faculty of Pharmacy, Srinakharinwirot University Ethics Committees, no. $012 / 2014$ ) and were free to withdraw at any time. Subjects did not show any symptoms of upper respiratory infections. The odor preference test was done by ranking test. The odor acceptance test was done by a hedonic test. Consumers sniffed four products, namely BEOP1, BEOP2, BEOP3, and BEOP4. To minimize sensory adaptation, consumers sniffed the coffee beans between each product (Moon and Li-Chan, 2007). Consumers ranked of the preference for those products. Odor preference choices were 1-the most; 2-very; 3-moderate; 4-least. Consumers gave a rating of acceptance for those products. The 9-point hedonic scale was used in the acceptance test. Select the good BEOP which consumers give the most preference and the most acceptance to further develop the preparation in the next step.

\section{Formulation of carier oil formulas (COFs)}

Three carrier oil formulas (COFs) were prepared in a volume ratio, as shown in Table 2. COF1 was composed of sweet almond: apricot: avocado: jojoba oils (10:8:1:1). COF2 
was composed of sweet almond: grape seed: avocado: jojoba oils (8:10:1:1). COF3 was composed of sweet almond: grape seed: avocado: macadamia oils (10:8:1:1). COF1 prepared as following: pipette $10 \mathrm{~mL}$ of almond, $8 \mathrm{~mL}$ of apricot, $1 \mathrm{~mL}$ of avocado, and $1 \mathrm{~mL}$ of jojoba oils in a light-resistant bottle. Then close tightly and shake well until homogeneous. Kept in a cool place until use. Other COFs were prepared in the same procedure as COF1 but changing the amount and type of the carrier oils. Three COFs were evaluated consumers' satisfaction.

Table 2: Carrier oil formulas (COFs).

\begin{tabular}{cccc}
\hline Preparation $(\mathrm{mL})$ & COF1 & COF2 & COF3 \\
\hline Almond & 10 & 8 & 10 \\
Grapeseed & - & 10 & 8 \\
Apricot & 8 & - & - \\
Avocado & 1 & 1 & 1 \\
Jojoba & 1 & 1 & - \\
Macadamia & - & - & 1 \\
\hline
\end{tabular}

$\mathrm{COF} 1=$ carrier oil formula $1, \mathrm{COF} 2=$ carrier oil formula $2, \mathrm{COF} 3=$ carrier oil formula 3 .

\section{Evaluation of consumers' satisfaction}

Three COFs were evaluated consumers' satisfaction. Thirty consumers participated in the study. They were fully briefed, given written informed consent to all aspects of the study (Faculty of Pharmacy, Srinakharinwirot University Ethics Committees, no. 005/M2016) and were free to withdraw at any time. Subjects did not show any symptoms of upper respiratory infections. Evaluation of consumers' satisfaction was done by questionnaire (Koonlaboot and Hongratanaworakit, 2015). The questionnaire was verified by experts in terms of content validity, idioms, forms, and the overall contents. The Internal Consistency Reliability was at an acceptable level. The Cronbach's Alpha Coefficient was 0.775. The satisfaction of the products was evaluated in terms of ability to spread on the skin, ability to penetrate the skin, skin hydration, softness of the skin, greasy of the skin, oil on the skin, and overall formulas satisfaction. The scores for ability to spread on the skin, ability to penetrate the skin, skin hydration, and softness of the skin were 1-least satisfaction to 5-most satisfaction. While the scores for greasy of the skin and oil on the skin were 1-very, 2-fit, and 3-little. The score for satisfaction with overall formulas was 10 points. Select the good COF which consumers give the most satisfaction to further develop the preparation in the next step.

\section{Formulation of massage oil formulas}

Carrier oils have low stability and low absorption through the skin. Therefore, use of these oils in the product should add an antioxidant to reduce rancidity and improve the stability of the product. Synthetic triglycerides, i.e., isopropyl myristate, isopropyl palmitate, and isopropyl laurate, were used to solve the problem of rancid oils. These compounds had saturated triglycerides, which had a good stability. In this study, we selected isopropyl myristate because it had low viscosity, high spread on the skin, good absorb, high dissolve into the carrier oil, and odorless. In addition, vitamin $\mathrm{E}$ acetate used as an antioxidant in the product. Isopropyl myristate was added into the massage oil formulas and used in the range $10-30 \%$ by volume. Massage oils were prepared in a volume ratio, as shown in Table 3. F1 prepared as following: add $96 \mathrm{~mL}$ of COF2 into a light-resistant bottle. Then add $4 \mathrm{~mL}$ of BEOP 1 and $0.05 \mathrm{~mL}$ of vitamin acetate. After that close tightly and shake well until homogeneous. Kept in a cool place until use. Other massage oil formulas were prepared in the same procedure as F1 but changing the amount and type of the base oils.

Table 3: Massage oil formulas for relieving muscle pain.

\begin{tabular}{cccccc}
\hline Formulas (mL) & BEOP1 & Vit E & COF2 & COF3 & IPM \\
\hline F1 & 4 & 0.05 & 96 & - & - \\
F2 & 4 & 0.05 & - & 96 & 9.6 \\
F3 & 4 & 0.05 & 86.4 & - & 9.6 \\
F4 & 4 & 0.05 & - & 86.4 & 19.2 \\
F5 & 4 & 0.05 & 76.8 & - & 19.2 \\
F6 & 4 & 0.05 & - & 76.8 & 28.8 \\
F7 & 4 & 0.05 & 67.2 & - & - \\
F8 & 4 & 0.05 & - & 67.2 & 28.8 \\
\hline
\end{tabular}

Vit $\mathrm{E}=$ Vitamin $\mathrm{E}$ acetate, $\mathrm{IPM}=$ Isopropyl myristate, $\mathrm{COF} 2=$ carrier oil formula $2, \mathrm{COF} 3=$ carrier oil formula $3, \mathrm{BEOP} 1=$ blended essential oil preparation 1.

\section{Preliminary physical stability evaluation}

The products were evaluated the preliminary physical stability in terms of color, smell, rancidity, viscosity, and precipitate. The products were kept in closed containers and kept at room temperature about $25-30^{\circ} \mathrm{C}$ for a period of eight weeks. Physical characteristics were evaluated at $0,1,2,4,7$, and 8 weeks.

\section{Safety test}

Ten healthy volunteers took part in the safety test. They were fully briefed, given written informed consent to all aspects of the study (Faculty of Pharmacy, Srinakharinwirot University Ethics Committees, no. 005/M2016) and were free to withdraw at any time. A Kligman maximization method (Waggoner, 1990) was used to evaluate skin irritation and skin sensitization. The subject's arm side was randomly selected. Then $0.1 \mathrm{~mL}$ of the product or the control was applied to the inner forearm and covered with a semi-occlusion patch (Tegaderm ${ }^{\circledR}$ tape) for 48 hours. After that, the patch was removed. This procedure was repeatedly performed in the next ten days. Clinical evaluation of skin irritation and skin sensitization was done using the visual scoring scale of the International Contact Dermatitis Research Group (ICDRG) as follows: 0 = negative reaction; 1 = weak reaction; $2=$ strong reaction; 3 = extreme reaction. Whenever weak reaction was found in subjects more than $10 \%$ of the subjects, the application was discontinued.

\section{Statistical analysis}

The SPSS version 21 was used for statistical analysis. The odor preference, odor acceptance, and satisfaction rating of products were analyzed by using a non-parametric Kruskal-Wallis test for comparison of all groups and using a non-parametric Mann-Whitney test for comparison between two groups. A p-value $<0.05$ was considered statistically significant. 


\section{RESULTS AND DISCUSSION}

\section{Odor preference test}

Odor preference ratings for four BEOPs are presented in Table 4. Consumers gave the most preference to BEOP1. Comparison of the odor preference ratings of four BEOPs showed significant differences in all groups $(p<0.05)$. The odor preference rating of the BEOP 1 was significantly higher than that of BEOP 2 , 3 , and $4(\mathrm{p}<0.001)$.

Table 4: Odor preference ratings for four BEOPs $(n=61)$.

\begin{tabular}{ccccc}
\hline \multirow{2}{*}{ Preparation } & \multicolumn{4}{c}{ Odor preference ratings (frequency) } \\
\cline { 2 - 5 } & The most & Very & Moderate & Least \\
\hline BEOP 1 & 25 & 21 & 11 & 4 \\
BEOP 2 & 10 & 12 & 22 & 17 \\
BEOP 3 & 11 & 14 & 19 & 17 \\
BEOP 4 & 15 & 14 & 9 & 23 \\
\hline
\end{tabular}

\section{Odor acceptance test}

Odor acceptance scores for four BEOPs are presented in Table 5. Comparison of the odor acceptance scores of four BEOPs showed significant differences in all groups $(p<0.05)$. The odor acceptance rating of the BEOP 1 was significantly higher than that of BEOP 2, 3, and $4(\mathrm{p}<0.001)$.

Table 5: Odor acceptance score for four BEOPs $(n=61)$.

\begin{tabular}{cc}
\hline Preparation & Mean acceptance score \pm SD \\
\hline BEOP 1 & $6.64 \pm 1.57$ \\
BEOP 2 & $5.41 \pm 2.21$ \\
BEOP 3 & $5.80 \pm 2.09$ \\
BEOP 4 & $5.39 \pm 2.19$ \\
\hline
\end{tabular}

In conclusion, BEOP 1 is a preparation that consumers have the most preference and the most acceptance, therefore, this formula was selected to develop the preparation for the next step.

\section{Evaluation of consumers' satisfaction}

Three carrier oil formulas (COFs) were evaluated consumers' satisfaction. Thirty consumers participated in the study. The satisfaction of the COFs was evaluated by questionnaire in terms of ability to spread on the skin, ability to penetrate the skin, skin hydration, softness of the skin, greasy of the skin, oil on the skin, and overall formulas satisfaction. The satisfaction ratings for three COFs are presented in Table 6.

Table 6: The satisfaction ratings for three COFs.

\begin{tabular}{lccc}
\hline & \multicolumn{3}{c}{ Mean \pm SD } \\
\cline { 2 - 4 } & COF1 & COF2 & COF3 \\
\hline Ability to spread on the skin* & $3.50 \pm 0.90$ & $3.87 \pm 0.82$ & $3.80 \pm 0.76$ \\
Ability to penetrate the skin* & $2.77 \pm 0.73$ & $3.63 \pm 0.93$ & $3.67 \pm 0.92$ \\
Skin hydration* & $3.87 \pm 0.57$ & $4.03 \pm 0.49$ & $3.83 \pm 0.65$ \\
Softness of the skin* & $3.47 \pm 0.57$ & $3.67 \pm 0.55$ & $3.77 \pm 0.73$ \\
Greasy of the skin** & $1.30 \pm 0.47$ & $1.80 \pm 0.55$ & $2.07 \pm 0.74$ \\
Oil on the skin** & $1.13 \pm 0.35$ & $1.57 \pm 0.63$ & $1.90 \pm 0.80$ \\
Overall formulas satisfaction**** & $6.38 \pm 1.54$ & $7.53 \pm 1.22$ & $7.58 \pm 1.74$ \\
\hline
\end{tabular}

*The scores: 1-least satisfaction to 5 -most satisfaction, $* *$ the scores: 1-very 2 -fit 3 -little, $* * *$ satisfaction with overall formulas score of 10 points.
The results showed that consumers gave an overall satisfied with COF1 less than COF2, COF3, as shown in Table 6. The COF1 had the ability to spread on the skin, ability to penetrate the skin, and softness of the skin less than the COF2 and COF3. The COF2 gave the highest skin hydration. Moreover, COF1 made sticky and oily skin too much. Considering the overall average satisfaction between COF2 and COF3 seems no difference, therefore, the statistical analysis of data should be done in order to choose the best formula, as shown in Table 7. Comparison of the consumers' satisfaction of three COFs showed significant differences in terms of ability to penetrate the skin $(p=0.000)$, greasy on the skin $(p=0.000)$, oil on the skin $(p=$ $0.000)$, and overall formulation satisfaction $(p=0.005)$. While no significant difference in terms of the ability to spread on the skin, skin hydration, softness of the skin was found $(p>0.05)$.

Table 7: The statistical analysis of all COF groups for satisfaction ratings.

\begin{tabular}{lcc}
\hline & \multicolumn{2}{c}{ COF1-COF2-COF3 } \\
\cline { 2 - 3 } & Chi-Square & Asymp. Sig. \\
\hline Ability to spread on the skin & 3.053 & 0.217 \\
Ability to penetrate the skin & 17.456 & $0.000^{*}$ \\
Skin hydration & 2.245 & 0.325 \\
Softness of the skin & 3.969 & 0.137 \\
Greasy of the skin & 19.884 & $0.000^{*}$ \\
Oil on the skin & 18.239 & $0.000^{*}$ \\
Overall formulas satisfaction & 10.683 & $0.005^{*}$ \\
\hline
\end{tabular}

$* \mathrm{p}<0.05, \mathrm{n}=30$.

Comparison of the consumers' satisfaction between COF groups was presented in Table 8. COF2 and COF3 had the ability to penetrate the skin, greasy of the skin, oil on the skin, and overall formulas satisfaction better than COF1 $(\mathrm{p}<0.05)$. While no significant difference in terms of the ability to spread on the skin, skin hydration, softness of the skin was found $(\mathrm{p}>0.05)$. However, consumers' satisfaction with COF2 and COF3 showed no significant differences. In conclusion, we selected COF2 and COF3 to develop the product in the next step.

Table 8: The statistical analysis between COF groups for satisfaction ratings.

\begin{tabular}{lccc}
\hline & \multicolumn{3}{c}{ Asymp.Sig } \\
\cline { 2 - 4 } & COF1/COF2 & COF1/COF3 & COF2/COF3 \\
\hline Ability to spread on the skin & 0.110 & 0.179 & 0.717 \\
Ability to penetrate the skin & $0.000^{*}$ & $0.000^{*}$ & 0.797 \\
Skin hydration & 0.221 & 0.791 & 0.166 \\
Softness of the skin & 0.144 & 0.066 & 0.530 \\
Greasy of the skin & $0.001 *$ & $0.000^{*}$ & 0.132 \\
Oil on the skin & $0.002 *$ & $0.000^{*}$ & 0.105 \\
Overall formulas satisfaction & $0.005^{*}$ & $0.005^{*}$ & 0.612 \\
\hline
\end{tabular}

${ }^{*} \mathrm{p}<0.05, \mathrm{n}=30$

\section{Preliminary physical stability evaluation}

The developed massage oils were evaluated the preliminary physical stability in terms of color, smell, rancidity, viscosity, and a precipitate. Color, viscosity, precipitate of all formulas were not changed for 8 weeks. While the smell of 
products had faded since the fourth week except for F1 and F6. The rancidity of products increased since the seventh week except for F1 and F6. Therefore, F1 and F6 were selected to test of product safety in the next step.

\section{Safety test}

The skin irritation and skin sensitization of the products were tested by using the Kligman maximization method (Waggoner, 1990). The result showed that no skin irritation and no skin sensitization were observed after volunteers applied F1 and F6 on the inner forearm for two weeks. Therefore, it could be suggested that both F1 and F6 were safety.

\section{CONCLUSIONS}

In conclusion, a massage oil formula which composed of BEOP1 (rosemary, lavender, patchouli, eucalyptus, and peppermint oils), vitamin $\mathrm{E}$ acetate, isopropyl myristate, and carrier oils (sweet almond, grape seed, avocado, jojoba oils, and macadamia oils) gave the best physical characteristics. Moreover, developed massage oil remained stable for 8 weeks. No skin irritation and no skin sensitization were observed after volunteers applied to developed massage oil on the inner forearm for two weeks. Therefore, it could be suggested that developed massage oil was safety. These findings indicate the possibility to use massage oil formulas for an alternative product for relieving muscle pain, but there should be further investigate the efficacy of these products in patients to obtain the complete results.

\section{ACKNOWLEDGEMENTS}

This work was supported by grants from Srinakharinwirot University (063/2016). The authors are grateful to the people who participated in the study.

\section{REFERENCES}

Albert YL, Steven F. 1996. Encyclopedia of common natural ingredient 2nd Ed. John Wiley \& Sons Inc., New York, United States.

Cooksley V. 1996. Aromatherapy: a lifetime guide to healing with essential oils. Prentice Hall, New Jersey, United States.

Damain P, Damain K. 1995. Aromatherapy: scent and psyche: using essential oils for physical and emotional well-being. Healing Arts Press, Vermont, United States.

Gobel H, Schmid G, Soyka D. Effect of peppermint and eucalyptus oil preparations on neurophysiological and experimental algesimetric headache parameters. Cephalalgia. 1994; 14:228-234.

Golab M, Skwarlo-Sonta K. Mechanisms involved in the antiinflammatory action of inhaled tea tree oil in mice. Exp Biol Med. 2007; 232:420-426.

Hajhashemi V, Ghannadi A, Jafarabadi H. Black cumin seed essential oil, as a potent analgesic and anti-inflammatory drug. Phytother Res. 2004; 18:195-199.

Hong CZ, Shellock FG. Effects of a topically applied counterirritant (Eucalyptamint) on cutaneous blood flow and on skin and muscle temperatures. A placebo-controlled study. Am J Phys Med Rehab. 1991; 70:29-33.

Hudson CM. 1999. Aromatherapy massage. Dorling Kindersley Limited, London, UK.

Juergens UR, Stober M, Vetter H. Inhibition of cytokine production and arachidonic acid metabolism by eucalyptol (1,8-cineole) in human blood monocytes in vitro. Eur J Med Res. 1998a; 3:508-510.

Juergens UR, Stober M, Schmidt-Schilling L, Kleuver T, Vetter H. Anti-inflammatory effects of eucalyptol (1,8-cineole) in bronchial asthma inhibition of arachidonic acid metabolism in human blood monocytes ex vivo. Eur J Med Res. 1998b; 3:407-412.

Koonlaboot M, Hongratanaworakit T. Muscle pain relief effect and satisfaction of blended essential oil preparation. Srinakharinwirot University Journal of Science and Technology. 2015; 7:50-64.

Larry CFL, Orth M, Ricky WK. Clinical effect of a topical herbal ointment on pain in temporo-mandibular disorders: a randomized placebo-controlled trial. J Altern Complement Med. 2009; 15:1311-1317.

Lawless J. 1999. The Illustrated Encyclopedia of Essential Oils: The Complete Guide to the Use of Oils in Aromatherapy and Herbalism. Element Book Limited, Boston, United States.

Lis-Balchin M. 2006. Aromatherapy science: a guide for healthcare professionals. Pharmaceutical Press, London, England.

Moon SY, Li-Chan CY. Changes in aroma characteristics of stimulated beef flavor by soy protein isolate assessed by descriptive sensory analysis and gas chromatography. Food Res Int. 2007; 40:1239-1248.

Narishetty ST, Panchagnula R. Effect of L-menthol and 1,8-cineole on phase behavior and molecular organization of SC lipids and skin permeation of zidovudine. J Control Release. 2005; 102:59-70.

Price S, Price L. 2003. Aromatherapy for Health Professionals, 2nd ed. Churchill Livingstone, New York, United States.

Romano TJ, Stiller JW. Usefulness of topical methyl salicylate, camphor, and menthol lotion in relieving pain in fibromyalgia syndrome patients. AJPM. 1994; 4:172-174.

Rutledge DN, Jones CJ. Effects of topical essential oil on exercise volume after a 12-week exercise program for women with fibromyalgia: a pilot study. J Altern Complement Med. 2007; 13:1099-1106.

Silva J, Abebe A, Sousa SM, Duarte VG, Machado MIL, Matos FJA. Analgesic and anti-inflammatory effects of essential oils of Eucalyptus. J Ethnopharmaco. 2003; 89:277-283.

Tekeoglu I, Dogan A, Demiralp L. Effects of thymoquinone (volatile oil of black cumin) on rheumatoid arthritis in rat models. Phytother Res. 2006; 20:869-871.

Waggoner WC. 1990. Clinical safety and efficacy testing of cosmetics. Marcel Dekker Inc., New York, United States. London, UK.

Wildwood C. 2000. Aroma remedies. Collins \& Brown Limited,

How to cite this article:

Hongratanaworakit T, Soontornmanokul S, Wongareesanti P. Development of Aroma Massage Oil for Relieving Muscle Pain and Satisfaction Evaluation in Humans. J App Pharm Sci, 2018; 8(04): 126-130. 